\title{
Immunosuppression of breast cancer cells mediated by transforming growth factor- $\beta$ in exosomes from cancer cells
}

\author{
LEI RONG, RONG LI, SHAOYING LI and RONGCHENG LUO
}

\author{
Department of Oncology, Nanfang Hospital, Nanfang Medical University, Guangzhou, Guangdong 510515, P.R. China
}

Received March 30, 2014; Accepted December 16, 2015

DOI: $10.3892 / 01.2015 .3841$

\begin{abstract}
Exosomes derived from tumor cells are essential for processes involved in tumor progression, including angiogenesis, tumor cell proliferation and immunoregulation. In addition, exosome secretion may contribute to the mechanisms of hypoxia-induced angiogenesis and metastasis of tumors. In the present study, as it is one of the most common cancers in females, breast cancer, cell lines were cultured under hypoxic $\left(1 \% \mathrm{O}_{2}\right)$ and normoxic conditions to evaluate the effects of hypoxia on exosome production. Under hypoxic conditions an increase in the number of exosomes in the medium, determined by CD63 immunoblotting, was observed. Application of these exosomes to T cells revealed that they were able to suppress $\mathrm{T}$ cell proliferation. As transforming growth factor- $\beta$ (TGF- $\beta$ ), interleukin-10, and prostaglandin E2 are important factors in the mediation of $\mathrm{T}$ cell suppression, the exosomes were subsequently treated with antibodies against these three factors. The results revealed that anti-TGF- $\beta$ was capable of ameliorating the immunosuppressive effects of exosomes. These data demonstrate that hypoxia enhances the secretion of exosomes by breast cancer cells, which acts to suppress $\mathrm{T}$ cell proliferation via TGF- $\beta$. The findings have significant implications for understanding the underlying mechanisms of immunosuppression in tumor microenvironments, and for the potential development of cancer therapies.
\end{abstract}

\section{Introduction}

The communication dysfunction between tumor cells and stromal cells is essential for tumorigenic processes, including cellular proliferation, differentiation, and apoptosis (1). Tumor cells must evade and/or suppress immune responses locally. As chronic inflammation is an important feature of the tumor microenvironment (1), various immune cells infiltrate the

Correspondence to: Dr Rongcheng Luo, Department of Oncology, Nanfang Hospital, Nanfang Medical University, 1838 North Guangzhou Avenue, Guangzhou, Guangdong 510515, P.R. China

E-mail: rongchengluo@126.com

Key words: hypoxia, tumor, migration, breast cancer cells, exosomes tumor tissue, including $\mathrm{T}$ cells, $\mathrm{B}$ cells, macrophages, and dendritic cells. These immune cells interact with tumor cells, stroma cells and each other, constituting a complex tumor microenvironment, however, the immune response remains weak, even in the presence of chronic inflammation.

Recently, exosomes have emerged as potent communicators between tumors and their microenvironment, and potential contributors to tumorigenesis and subsequent metastasis $(2,3)$. Exosomes are nanoparticles of 50-100 $\mathrm{nm}$ in size, and are reported to be derived from endosomes or multivesicular bodies. Exosomes are present in the extracellular microenvironment and in bodily fluids including blood and urine $(4,5)$.

Accumulating research indicates that exosomes have diverse functions in an array of biological events, such as cellular proliferation and migration (6). The contents of exosomes are complex and may include diverse RNAs, cytokines, growth factors and lipids (7-10), which are specific to their function and indicate their biological significance. Exosomes contain selectively enriched mRNAs and miRNAs that regulate gene expression in target cells, including antigen-presenting cells; cell-derived exosomes act to induce an immune response, whereas tumor-derived exosomes act to suppress it

The essential roles of exosomes have been demonstrated in a number of studies conducted in various tumor types, such as prostate cancer, and melanoma $(9,11,12)$. Exosomes derived from different types of cancer contain different combinations of biological molecules, and have distinctive effects on tumor behavior. Many miRNAs have been discovered in exosomes from tumor cells and stroma cells, which are essential in the regulation of tumor cell migration $(9,10,12-16)$. Certain miRNAs may be involved in multiple stages of tumor development; for example, miR-31, -185 , and $-34 \mathrm{~b}$ are involved in melanoma invasion (10). Therefore, exosomes have been proposed to be potential markers for the prognosis and diagnosis of various cancer types, and may be potential therapeutic targets $(9,13,14)$.

Lymphocyte infiltration and activation in tumor microenvironments may have a significant effect on tumor prognosis, growth and metastasis $(5,17,18)$. It has been hypothesized that well-established cancer cells utilize multiple pathways to evade and/or suppress the immune response against tumors (18); manipulating the underlying mechanisms of this immune response has therefore been the focus of many therapies for the treatment of various types of cancer.

Breast cancer is one of the most common cancers in females, and one of the greatest threats to female health. 
Globally, breast cancer was the cause of 522,000 mortalities (15\% of cancer mortalities in females) in 2012 (19). Rapid progress in medical treatment has markedly improved the prognosis and diagnosis, however, surgery remains the most effective treatment, which often has a significant physiological and psychological impact on patients $(20,21)$. Elucidation of the mechanisms underlying tumorigenesis in breast cancer would aid in the development of treatments for this condition. Although certain studies have indicated the involvement of cancer cell-derived exosomes in tumor growth, few have explored the role of exosomes in the development of breast cancer $(22,23)$.

The present study explores the immunoregulatory functions of exosomes derived from two different breast cancer cell lines (MDA-MB-231 and BT-474). As transforming growth factor- $\beta$ (TGF- $\beta$ ) and interleukin-10 (IL-10), the two predominantly considered cytokines, have been demonstrated to suppress the immune response $(24,25)$, the presence of these factors in the exosomes from these cell lines was also investigated.

\section{Materials and methods}

Cell culture and transfection. BT-474 and MDA-MB-231 breast cancer cells were purchased from American Type Culture Collection (Manassas, VA, USA), and cultured according to the supplier's instructions. The cells were cultured in Dulbecco's modified Eagle's medium (DMEM; Invitrogen Life Technologies, Carlsbad, CA, USA) supplemented with $10 \%$ fetal bovine serum (FBS). When the cells were $\sim 50 \%$ confluent, the medium was changed to serum-free medium prior to exosome preparation.

Hypoxic exposure. For hypoxic exposure, cells were cultured in an $\mathrm{O}_{2} / \mathrm{CO}_{2}$ incubator (Sanyo; Osaka, Japan) in a $1 \%$ $\mathrm{O}_{2}, 5 \% \mathrm{CO}_{2}$ humidified atmosphere at $37^{\circ} \mathrm{C}$. For certain experiments, cells were treated with dimethyloxalylglycine, a hypoxia-inducible factor hydroxylase inhibitor (Enzo Life Sciences; Farmingdale, NY, USA) at a final concentration of $1 \mathrm{mM}$. This was added to inhibit the effects of hypoxia.

Normoxic exposure. For normoxic exposure, cells were cultured in an $\mathrm{O}_{2} / \mathrm{CO}_{2}$ incubator (Sanyo) in a $20 \% \mathrm{O}_{2}, 5 \%$ $\mathrm{CO}_{2}$ humidified atmosphere at $37^{\circ} \mathrm{C}$.

Exosome preparation. BT-474 and MDA-MB-231 cell lines were cultured in serum-free medium for exosome preparation. Exosome preparation was performed as described by Epple et al (26) with slight modification. After four days of culture, $100 \mathrm{ml}$ of media were collected. Exosomes were harvested through serial centrifugation of supernatants $(2 \times 10 \mathrm{~min}, 500 \times \mathrm{g} ; 1 \times 20 \mathrm{~min}, 2,000 \mathrm{x} \mathrm{g} ; 1 \times 30 \mathrm{~min}$, $10,000 \mathrm{x} \mathrm{g})$, followed by centrifugation $(60 \mathrm{~min}, 100,000 \mathrm{x} \mathrm{g})$. The resulting pellet was resuspended in $4 \mathrm{ml}$ phosphate buffered saline (PBS), and harvested again at 100,000 x g, $60 \mathrm{~min}$. The final exosome-containing pellet was resuspended in $0.4 \mathrm{ml}$ of PBS and quantified. For quantification, the resulting pellet was subjected to BCA assay using BCA protein assay kit (Pierce, Rockford, IL, USA). The CD63 expression was also detected using western blotting, which
A

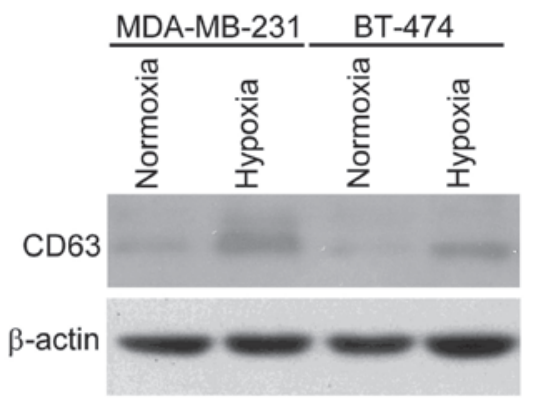

B

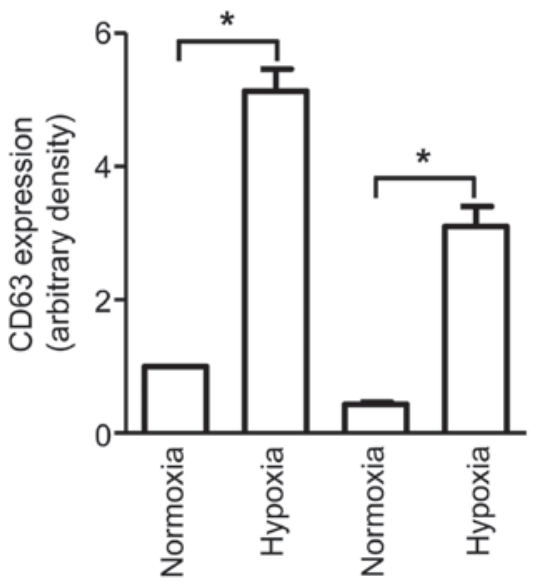

Figure 1. Exosomes were collected from MDA-MB-231 and BT- 474 cells cultured under normoxic or hypoxic conditions and lysed with $1 \%$ Nonidet P-40. Following SDS-PAGE, immunoblotting and exposure to detection reagents, relative densitometry was performed, which revealed higher expression of CD63 protein for cells cultured under hypoxic conditions compared with normoxic conditions for both cell lines. (A) Immunoblot showing increased CD63 expression in MDA-MB-231 and BT-474 cells cultured under hypoxic conditions. (B) Relative CD63 expression. For both cell lines, cells cultured in hypoxic conditions exhibited significantly higher CD63 protein levels than those cultured in normoxic conditions. Bars represent mean \pm standard error of the mean numbers of transmigrated cells from three independent experiments. ${ }^{*} \mathrm{P}<0.05$

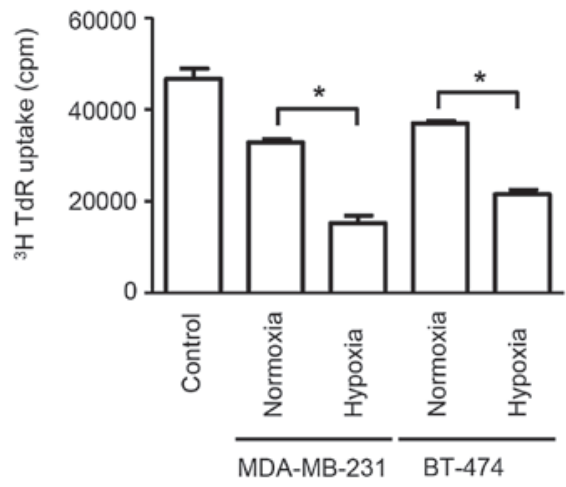

Figure 2. Splenocytes incubated with breast cancer cell-derived exosomes exhibit lower $\left[{ }^{3} \mathrm{H}\right]$ thymidine incorporation compared with controls. Additionally, splenocytes incubated with exosomes produced under hypoxic conditions exhibit significantly lower incorporation compared with those produced under normoxic conditions for the cancer cell lines, MDA-MB-231 and BT-474. Bars represent mean \pm standard error of the mean numbers of thymidine incorporation (cpm) from three independent experiments. ${ }^{*} \mathrm{P}<0.05$. $\mathrm{TdR}$, radioactive thymidine.

was normalized based on the cell number. Exosome preparations were stored at $-20^{\circ} \mathrm{C}$ until use. 
A

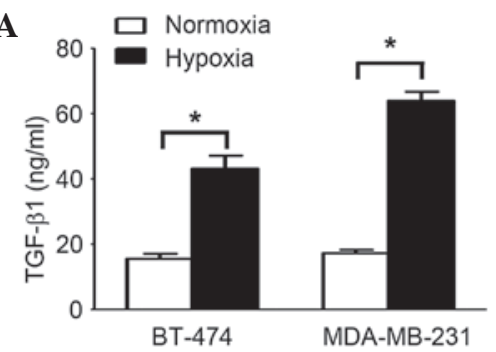

B

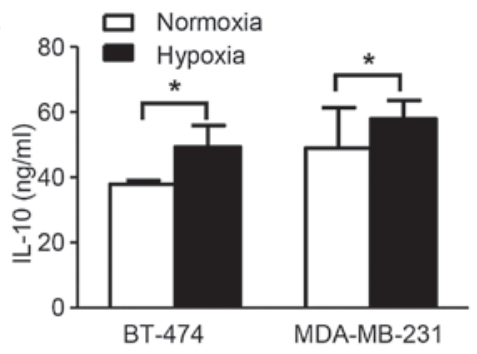

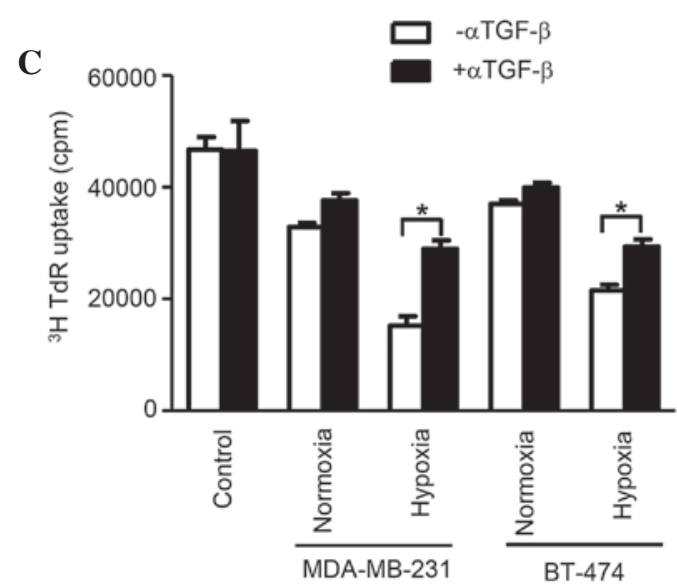

Figure 3. TGF- $\beta$ and IL-10 in exosomes are responsible for immunosuppression. Concentrations of (A) TGF- $\beta$ and (B) IL-10 in exosomes from MDA-MB-231 and BT-474, cultured under normoxia or hypoxia was measured by ELISA assay. (C) Addition of anti-TGF- $\beta$ antibodies was revealed to significantly reduce the effects of exosomes on $\left[{ }^{3} \mathrm{H}\right]$ thymidine uptake by proliferating splenocytes. Bars represent mean \pm standard error of the mean numbers of transmigrated cells from three independent experiments. " $\mathrm{P}<0.05$. TGF- $\beta$, transforming growth factor- $\beta$; IL-10, interleukin-10; TdR, radioactive thymidine.

Western blot analysis. Exosomes were collected and lysed with $1 \%$ Nonidet P-40 plus protease inhibitor cocktail (Roche, Indianapolis, IN, USA). Following brief lysis, $5 \mu \mathrm{g}$ exosome was subjected to $12 \%$ SDS-PAGE and transferred to nitrocellulose membranes. In this study, CD63 was used as an indicator for exosomes, as CD63 is a exosome marker. After blocking with $5 \%$ fat-free milk for $60 \mathrm{~min}$, the membrane was incubated with the appropriate primary antibody (CD63; Santa Cruz Biotech, Santa Cruz, CA, USA; catalog no. sc-15363), followed by a horseradish peroxidase-conjugated secondary antibody (Jackson ImmunoResearch, West Grove, PA, USA; catalog no. 111-035-003). $\beta$-actin was used as the loading control. The blotting bands were visualized with enhanced chemiluminescence plus immunoblotting detection reagents (Pierce, Rockford, IL, USA). The membrane was subsequently scanned and the relative densitometry of the bands was determined using ImageJ (Windows version 1.48, NIH, Bethesda, MD, USA).

Splenocyte proliferation assay. Splenocytes were isolated from the spleens of female C57BL/6 mice (6-8 weeks of age). The mice were purchased from the Shanghai Laboratory Animal Center of the Chinese Academy of Sciences (Shanghai, China), and all animal experiments were approved by the Institutional Review Board of Nanfang Medical University (Guangzhou, Guangdong, China). Splenocytes were separated and seeded at a density of $5 \times 10^{3}$ cells/well in DMEM supplemented with 10\% FBS. Anti-CD3 (Invitrogen
Life Technologies; catalog no. 11452D) and anti-CD28 (Invitrogen Life Technologies; catalog no. 11452D) linked with goat-anti-mouse antibody were added for activation. Splenocytes at a density of $5 \times 10^{4}$ cells/well in $100 \mu \mathrm{l}$ expansion medium were added to round-bottom 96-well plates (Nunc; Penfield, NY, USA) and cultured for five days. Activated splenocytes without addition of any exosomes were used as the control group. The immunosuppressive effects of the exosomes were evaluated by incubating $10 \mu \mathrm{l}$ exosomes per well with the splenocytes for five days. $\left[{ }^{3} \mathrm{H}\right]$ thymidine $(1 \mu \mathrm{Ci}$ $\mathrm{ml}^{-1}$; Perkin Elmer, San Jose, CA, USA) was added $18 \mathrm{~h}$ prior to the end of incubation. Cells were subsequently harvested for measurement of $\left[{ }^{3} \mathrm{H}\right]$ thymidine incorporation to assess their rate of proliferation. To harvest the cells, $1 \mathrm{ml} \mathrm{10 \%}$ trichloroacetic acid (TCA) was gently added to each well, and incubated at room temperature for $15 \mathrm{~min}$. Following aspiration with $10 \%$ TCA and washing with a further $1 \mathrm{ml}$ $10 \%$ TCA, the TCA was aspirated and $300 \mu 10.2 \mathrm{~N} \mathrm{NaOH}$ was added to each well to dissolve DNA; this was incubated for $15 \mathrm{~min}$ at room temperature with agitation. To measure the thymidine incorporation, subsequently, $100 \mu \mathrm{l}$ of each sample was added to a scintillation vial, and the counts were measured in a scintillation counter (Beckman Coulter LS6500; Brea, CA, USA). In order to assess the effects of TGF- $\beta$ and IL-10 on splenocyte proliferation, anti-TGF- $\beta$ and/or anti-IL-10 antibodies $(5 \mu \mathrm{g} / \mathrm{ml})$ were applied at the time of exosome application to block the potential effects of these factors. 
ELISA. The concentrations of TGF-b and IL-10 from exosome lysates were determined by ELISA according to the manufacturer's instructions (R\&D, Minneapolis, MN, USA; DB100B and HS100C, respectively). Briefly, $100 \mu \mathrm{l} /$ well exosome lysate samples were added to 96 -well plates and incubated for $2 \mathrm{~h}$ at room temperature. Following three washes with washing buffer (R\&D), $100 \mu \mathrm{l} /$ well polyclonal horseradish peroxidase-conjugated anti-TGF-b1 or monoclonal alkaline phosphatase-conjugated anti-IL-10 antibody was added and the samples were incubated at room temperature for $2 \mathrm{~h}$. The samples were then washed three times with wash buffer, $50 \mu \mathrm{l}$ substrate solution (R\&D) was added and incubated for $30 \mathrm{~min}$, followed by the addition of $50 \mu 1$ stop solution (R\&D). Absorbance was measured using a microplate reader (Biotek Synergy 2; Biotek, Winooski, VT, USA) at a wavelength of $490 \mathrm{~nm}$. The concentration of cytokines was quantified, according to a respective standard curve, which was obtained using recombinant human TGF-b1 or IL-10 as the control.

Statistical analysis. All statistical analyses were performed using SPSS software, version 20 (IBM SPSS Armonk, NY, USA). Student's $t$-test was used for comparison, and $\mathrm{P}<0.05$ was considered to indicate a statistically significant difference.

\section{Results}

Hypoxia increases exosome secretion from breast cancercells. Exosomes were isolated from the conditioned media of breast cancer cell lines BT-474 and MDA-MB-231, cultured under normoxic ( $21 \%$ oxygen) or hypoxic ( $1 \%$ oxygen) conditions, using serial centrifugation. The exosomal secretion of these cells was evaluated through immunoblotting using CD63, a well-established exosomal marker. As shown in Fig. 1, although MDA-MB-231 cells were observed to secrete more exosomes compared with BT-474 cells, both cell lines exhibited significantly higher CD63 protein levels for cells cultured in hypoxic conditions than for those cultured in normoxic conditions. This indicates that hypoxia is a potent inducer of exosome secretion, which may have a significant impact on the tumor microenvironment.

Exosomes derived from cancer cells suppress splenocyte proliferation. The application of exosomes to proliferating splenocytes revealed that exosomes secreted from cells cultured under hypoxic conditions produced significantly stronger immunosuppressive effects when compared with cells cultured under normoxic conditions $(\mathrm{P}<0.05)$. Additionally, stronger immunosuppressive effects were observed for exosomes secreted from cells in hypoxia than that from cells in normoxia $(\mathrm{P}<0.05)$. (Fig. 2). Exosomes derived from MDA-MB-231 cells under hypoxia exerted the most potent immunosuppression, followed by exosomes derived from BT-474 cells under hypoxia, MDA-MB-231 cells under normoxia and BT-474 under normoxia, which is consistent with the amount of exosome secretion observed on CD63 immunoblotting. These data demonstrate that exosomes from breast cancer cells cultured under hypoxic conditions have potent immunosuppressive effects, which potentially contribute to rapid tumor growth.
TGF- $\beta$ in exosomes is responsible for immunosuppression. The concentration of TGF- $\beta 1$ in exosomes from normoxic conditions in both cell lines was considerably higher compared with that from hypoxic conditions $(\mathrm{P}<0.05)$. Additionally, the concentration of IL-10 in exosomes from normoxic conditions in cell line BT-474 was significantly higher compared with hypoxic conditions, however, no considerable difference was observed for MDA-MB-231 cells. Furthermore, in BT-474 cells, the difference for TGF- $\beta$ is much more prominent than for IL-10 (Fig. 3A). The $\left[{ }^{3} \mathrm{H}\right]$ thymidine incorporation assay, which was applied to blocking TGF- $\beta 1$ activity using anti-TGF- $\beta$ antibodies, revealed that this factor is essential for immunosuppression, as demonstrated by the evaluation of splenocyte proliferation, which was restored following TGF- $\beta$ neutralization (Fig. 3B). Collectively, the results demonstrate that exosomes from breast cancer cells are able to suppress the immune response through TGF- $\beta$, which inhibits splenocyte proliferation.

\section{Discussion}

As exosomes contain bulky proteins, proteomic analysis has allowed for further investigation into the functions of exosomes. An array of proteins, including vascular endothelial growth factor, epidermal growth factor and interleukin-4, have been shown to be present in the exosomes from cancer cells, and are critical for tumor cell survival, proliferation and migration. TGF- $\beta 1$, which is produced by regulatory $\mathrm{T}$ cells, has potent immunosuppressive effects, which are crucial for protecting the body from excessive immune responses and maintaining immune homeostasis (27-29). However, these mechanisms may be manipulated by cancer cells, allowing them to evade and suppress immune responses. This study demonstrated that exosomes derived from breast cancer cells contain abundant TGF- $\beta$, which facilitates tumor growth. As hypoxia is a feature of the microenvironment of solid tumors, it is not surprising that the immunosuppressive effects of exosomes derived from cancer cells cultured under hypoxia are much greater than those of exosomes produced under normoxic conditions. This may explain the rapid growth of solid cancers in vivo in a hypoxic microenvironment. In the present study, it was demonstrated that hypoxia promoted the secretion of exosomes in cancer cells, which contain the immunosuppressive cytokines, TGF- $\beta$ and IL-10. It is hypothesized that exosomes are important for communication among cancer cells, and are able to fuse with adjacent cancer cells, subsequently increasing their immunosuppressive effects. Thus, we hypothesize that preventing communication among cancer cells may provide a novel method for the inhibition of cancer growth. However, as breast cancer cell lines were used in the present study the results may not be applicable in vivo. Therefore, further in vivo studies using animal models and clinical data are required. Additionally, the detailed mechanisms of exosome secretion and cancer cell communication also require further investigation.

In conclusion, these results revealed a novel aspect of exosomes derived from breast cancer cells in immunosuppression, suggesting the essential role of exosomes in tumor microenvironment constitution. These findings could be potentially significant in the development of novel therapeutic strategies. 


\section{References}

1. Hanahan D and Weinberg RA: Hallmarks of cancer: the next generation. Cell 144: 646-674, 2011.

2. Corrado C, Raimondo S, Chiesi A, Ciccia F, De Leo G and Alessandro R: Exosomes as intercellular signaling organelles involved in health and disease: basic science and clinical applications. Int J Mol Sci 14: 5338-5366, 2013.

3. Ahmed KA and Xiang J: Mechanisms of cellular communication through intercellular protein transfer. J Cell Mol Med 15: 1458-1473, 2011.

4. Denzer K, Kleijmeer MJ, Heijnen HF, Stoorvogel W and Geuze HJ: Exosome: from internal vesicle of the multivesicular body to intercellular signaling device. J Cell Sci 113 3365-3374, 2000.

5. Schorey JS and Bhatnagar S: Exosome function: from tumor immunology to pathogen biology. Traffic 9: 871-881, 2008.

6. van Niel G, Porto-Carreiro I, Simoes S and Raposo G: Exosomes: a common pathway for a specialized function. J Biochem 140: 13-21, 2006.

7. Bard MP, Hegmans JP, Hemmes A, et al: Proteomic analysis of exosomes isolated from human malignant pleural effusions. Am J Respir Cell Mol Biol 31: 114-121, 2004.

8. Street JM, Barran PE, Mackay CL, et al: Identification and proteomic profiling of exosomes in human cerebrospinal fluid. J Transl Med 10: 5, 2012.

9. Hessvik NP, Sandvig K and Llorente A: Exosomal miRNAs as Biomarkers for Prostate Cancer. Front Genet 4: 36, 2013.

10. Xiao D, Ohlendorf J, Chen Y, et al: Identifying mRNA, microRNA and protein profiles of melanoma exosomes. PloS One 7: e46874, 2012.

11. Díaz-Valdés N, Basagoiti M, Dotor J, et al: Induction of monocyte chemoattractant protein-1 and interleukin-10 by TGFbetal in melanoma enhances tumor infiltration and immunosuppression. Cancer Res 71: 812-821, 2011.

12. Mizoguchi M, Guan Y, Yoshimoto K, et al: Clinical implications of microRNAs in human glioblastoma. Front Oncol 3: 19, 2013.

13. Gailhouste L, Gomez-Santos L and Ochiya T: Potential applications of miRNAs as diagnostic and prognostic markers in liver cancer. Front Biosci (Landmark Ed) 18: 199-223, 2013.

14. Kahlert $\mathrm{C}$ and Kalluri R: Exosomes in tumor microenvironment influence cancer progression and metastasis. J Mol Med (Berl) 91: 431-437, 2013.

15. Katakowski M, Buller B, Zheng X, et al: Exosomes from marrow stromal cells expressing miR-146b inhibit glioma growth. Cancer Lett 335: 201-204, 2013
16. Chiba M, Kimura M and Asari S: Exosomes secreted from human colorectal cancer cell lines contain mRNAs, microRNAs and natural antisense RNAs, that can transfer into the human hepatoma HepG2 and lung cancer A549 cell lines. Oncol Rep 28: 1551-1558, 2012.

17. Borghesi L and Milcarek C: Innate versus adaptive immunity: a paradigm past its prime? Cancer Res 67: 3989-3993, 2007.

18. Lizée G, Overwijk WW, Radvanyi L, Gao J, Sharma P and Hwu P: Harnessing the power of the immune system to target cancer. Annu Rev Med 64: 71-90, 2013.

19. Tao Z, Shi A, Lu C, Song T, Zhang Z and Zhao J: Breast cancer: Epidemiology and etiology. Cell Biochem Biophys: Dec 28, 2014 (Epub ahead of print).

20. Murawa P, Murawa D, Adamczyk B and Połom K: Breast cancer: Actual methods of treatment and future trends. Rep Pract Oncol Radiother 19: 165-172, 2014

21. Edge SB: Quality measurement in breast cancer. J Surg Oncol 110: 509-517, 2014

22. Al-Nedawi K: The yin-yang of microvesicles (exosomes) in cancer biology. Front Oncol 4: 172, 2014

23. Tickner JA, Urquhart AJ, Stephenson SA, Richard DJ and O'Byrne KJ: Functions and therapeutic roles of exosomes in cancer. Front Oncol 4: 127, 2014

24. Jui HY, Lin CH, Hsu WT, et al: Autologous mesenchymal stem cells prevent transplant arteriosclerosis by enhancing local expression of interleukin-10, interferon- $\gamma$, and indoleamine 2,3-dioxygenase. Cell Transplant 21: 971-984, 2012.

25. Sun L, Akiyama K, Zhang H, et al: Mesenchymal stem cell transplantation reverses multiorgan dysfunction in systemic lupus erythematosus mice and humans. Stem Cells 27: 1421-1432, 2009.

26. Epple LM, Griffiths SG, Dechkovskaia AM, et al: Medulloblastoma exosome proteomics yield functional roles for extracellular vesicles. PloS One 7: e42064, 2012.

27. Vignali DA, Collison LW and Workman CJ: How regulatory T cells work. Nat Rev Immunol 8: 523-532, 2008.

28. Belkaid Y and Tarbell K: Regulatory T cells in the control of host-microorganism interactions(*). Annu Rev Immunol 27: 551-589, 2009

29. Zou W: Regulatory T cells, tumour immunity and immunotherapy. Nat Rev Immunol 6: 295-307, 2006. 